\title{
A Class of Hypercube-like Networks
}

\author{
Aniruddha S. Vaidya P. S. Nagendra Rao S. Ravi Shankar* \\ Department of Electrical Engineering, Indian Institute of Science, Bangalore 560012, India \\ asvaidya@ee.iisc.ernet.in
}

\begin{abstract}
We introduce a class of graphs which are variants of the hypercube graph. Many of the properties of this class of graphs are similar to that of the hypercube hence, we refer to them as the class of Hypercube-Like (HL) graphs/networks. We show that the hypercube, the twisted n-cube [1] and the multiply-twisted cube [2] are members of this class of graphs. We also propose simple strategies for distributed routing and broadcast and discuss some issues regarding embedding other graphs and reconfiguration in such networks.
\end{abstract}

\section{Introduction}

The attractive properties of the hypercube graph $[3]-$ a logarithmic diameter, a simple labeling scheme, good connectivity, recursive scalability and symmetry - have made it a popular topology for the design of multicomputer interconnection networks. Efforts to improve some of these properties have lead to the evolution of hypercube variants. Cube Connected Cycles, the Brigded and the Folded Hypercubes and the Extended Hypercube are variants (see [4] for references) derived through the addition of extra nodes and/or links to the hypercube. Another category of variants, which includes the Twisted n-cube [1] and the Multiply twisted cube [2], is derived by manipulating only the node-link incidences of the hypercube without the addition of extra nodes and links.

In this paper we present a general method of inductively constructing such a class of hypercube variants. We investigate the graph-theoretic properties of this class of networks. Since most of the properties of such graphs/networks ${ }^{1}$ are similar to that of the hypercube, we shall refer to them as Hypercube Like (HL) graphs/networks. We show that the hypercube, the twisted $n$-cube and the multiply twisted cube are members of this class. We propose simple strategies for distributed routing and broadcast in such networks and also discuss some aspects about reconfiguration.

For the sake of brevity, we omit all proofs and detailed discussions and provide only the key results and ideas. Detailed discussions and proofs of theorems are given in [4].

\footnotetext{
"Now with Tata Consultancy Services, Bangalore, India

"In the discussion to follow we shall use the terms "graphs" and "networks" interchangeably.
}

\section{Preliminaries}

\subsection{Notation}

Standard graph-theoretic notations [5] are used. Other terminology is explained below.

A permutation, $\pi^{n}$, is defined as a one-to-one correspondence on the object set $Z_{n}=\left\{0,1, \ldots, 2^{n}-1\right\}$. We use the one-line represenation for denoting a permutation.

The binary representation of a vertex labelled $v$ will be given by $\langle v\rangle_{2}$. Also, a string of $n$ bits, with $p$ leading 0 s will be represented as $0^{p} 1 *^{n-p-1}$ (' $*$ ' represents don't care).

The label of an edge of a graph is the number of significant bits in the XORed value of the binary representations of labels of its endpoints, i.e., for a graph $G$, the label of an edge $e\left(v_{i}, v_{j}\right)$ between two vertices $v_{i}$ and $v_{j} \in V(G)$, is given by, $\operatorname{label}\left(e_{v_{i} v_{j}}\right)=n-p$ where $p$ is derived from the $n$-bit binary representations of $v_{i}$ and $v_{j}$ from the relation $\left\langle v_{i}\right\rangle_{2} \oplus\left\langle v_{j}\right\rangle_{2}=0^{p} 1 *^{n-p-1}$. An edge or link ${ }^{2}$ with the label $k$ is called a $k$-link. Similarly, the dimension between any two vertices $v_{a}$ and $v_{b}$ (not necessarily adjacent) in $G$ is given by, $D I M\left(v_{a}, v_{b}\right)=n-p$, where $\left\langle v_{a}\right\rangle_{2} \oplus\left\langle v_{b}\right\rangle_{2}=0^{p} 1 *^{n-p-1}$.

The $n$-dimensional boolean hypercube ${ }^{3}$ graph, $Q_{n}$, is a $n$-regular labeled graph on $2^{n}$ vertices, the vertices being labeled 0 through $\left(2^{n}-1\right)$. Two vertices $v_{i}$ and $v_{j}$, belonging to $Q_{n}$, are connected by an edge if and only if the binary representations of their respective labels have a Hamming distance of 1. The 3-cube $\left(Q_{3}\right)$ is shown in Fig. 1(a).

\subsection{The class of HL-graphs}

The set of HL-graphs, a class of simple, connected, undirected graphs of dimension $n$ will be represented as $C H L_{n}$.

A graph $G_{n+1} \in C H L_{n+1}$ is inductively defined as:

$$
G_{n+1}=\operatorname{Inter}\left(G_{n}, G_{n}^{*}, \pi^{n}\right)
$$

where $G_{n}$ and $G_{n}^{*} \in C H L_{n}$, and the function Inter(.) which operates in the following two steps: step (i) interconnects the vertex $v_{i} \in V\left(G_{n}\right)$ to the vertex $\pi\left(v_{i}\right) \in V\left(G_{n}^{*}\right)$ by a edge, and step (ii) relabels the subset of vertices of $G_{n+1}$ corresponding to the vertices of $G_{n}^{*}$ by adding $2^{n}$ to their existing labels.

The basis of the inductive construction is $G_{0}$, the simple labelled graph on a single vertex labelled 0 .

\footnotetext{
"We shall use the terms "edge" and "link" interchangeably.

${ }^{3}$ The boolean hypercube has been variously referred to as the n-cube, binary hypercube or as the hypercube in the literature.
} 
Example 2.1 The construction of graph $G_{1}$ in Fig. 2 from $G_{0}$ is given by $G_{1}=\operatorname{Inter}\left(G_{0}, G_{0}, \pi^{0}\right)$, where $\pi^{0}(0)=0$. Similarly $G_{3}$ of Fig. 3 is constructed using $G_{2}$ and $G_{2}^{*}$ and $\pi^{2}=\{1,2,3,0\}$.

Since the function Inter(.) operates on two component graphs to give one resultant graph, the steps involved in the construction of an HL-graph can be represented as a binary tree. The binary tree like representation of the construction of the twisted 3-cube, $T Q_{3} \in C H L_{3}$, is shown in Fig. 4. The branches of the tree represent the constituent subgraphs of $T Q_{3}$ and the nodes represent the permutations used at the corresponding stages of the construction.

Using the binary tree like representation of the construction of an HL-graph, it is possible to enumerate the number of members belonging to the set of HL-graphs of a particular dimension i.e., determine its cardinality, $\left|C H L_{n}\right|$.

Theorem $2.1\left|C H L_{n}\right|=\prod_{i=0}^{n-1}\left[\left\{2^{n-i-1}\right\} !\right]^{2^{i}} ; n \geq 1$

Thus, the class of HL-graphs is extermely rich in terms of the number of members that belong to a particular dimension $-\left|C H L_{1}\right|=1,\left|C H L_{2}\right|=2,\left|C H L_{3}\right|=96$, and so on $\ldots$

\section{Topological properties}

We show that the members of this class have several properties which are similar to that of the binary hypercube. Some of these properties follow from the inductive construction of HL-graphs.

\subsection{Basic properties}

Theorem 3.1 The number of vertices in $a n$ dimensional $H L$-graph is $2^{n}$.

Theorem 3.2 The number of edges in a ndimensional $H L$-graph is $n 2^{n-1}$.

Theorem 3.3 An n-dimensional HL-graph is a $n$ regular simple graph.

Theorem 3.4 The number of $k$-links, $0<k \leq n$, in $a G_{n} \in C H L_{n}$ is $2^{n-1}$.

Theorem 3.5 No two edges incident on any vertex of a HL-graph have the same label.

Theorem 3.6 Connectivity of $G_{n} \in C H L_{n}$ is $n$.

Theorem 3.7 Given two distinct vertices, $v_{i}$ and $v_{j} \in V\left(G_{n}\right)$, there exist $n$ disjoint $v_{i}$ to $v_{j}$. paths in $G_{n} \in C H L_{n}$.

Theorem 3.8 A graph $G_{n} \in C H L_{n}$ iff

(a) $\left|V\left(G_{n}\right)\right|=2^{n}$

(b) $G_{n}$ is n-regular

(c) At every vertex, $v \in V\left(G_{n}\right)$, label $\left(e_{i}\right) \neq \operatorname{label}\left(e_{j}\right)$, for any two edges $e_{i}$ and $e_{j}$ incident at $v$ and $e_{i} \neq e_{j}$.

The above theorem permits one to identify networks which belong to the class of HL networks.

\section{Some known HL-graphs}

In this section we show that the $n$-cube, the twisted $n$ cube [1], and the multiply-twisted $n$-cube [2] belong to the class of HL-graphs.

\subsection{The $n$-cube}

The binary $n$-cube graph, $Q_{n}$, can be defined as follows:

$$
Q_{n}=\operatorname{Inter}\left(Q_{n-1}, Q_{n-1}, \pi_{I}^{n-1}\right)
$$

where, $\pi_{I}^{n-1}(x)=x$, for $0 \leq x \leq\left(2^{n-1}-1\right)$ and $Q_{0}$ is the same as $G_{0}$.

For a detailed discussion on the topological properties of $n$-cube see [3].

\subsection{The twisted $n$-cube}

The twisted $n$-cube can be defined as follows:

$$
T Q_{n}=\operatorname{Inter}\left(T Q_{n-1}, Q_{n-1}, \pi_{I}^{n-1}\right)
$$

where, $T Q_{2}$ is the basis for induction, and is defined as

$$
T Q_{2}=\operatorname{Inter}\left(Q_{1}, Q_{1},\{1,0\}\right) .
$$

The twisted $n$-cube is defined only for $n \geq 2$.

$T Q_{3}$ is shown in Fig. 1(b). It can be verified from the figure that $T Q_{3}$ has a diameter of only 2 as opposed to $Q_{3}$, which has a diameter of 3 . In general, a twisted $n$-cube, $T Q_{n}$, such that $n \geq 3$ has a diameter of $(n-1)$. A detailed discussion on the properties and optimal routing in twisted $n$-cube is given in [1].

\subsection{The multiply-twisted $n$-cube} lows:

The multiply-twisted cube $\left(M Q_{n}\right)$ can be defined as fol-

$$
M Q_{n}=\operatorname{Inter}\left(M Q_{n-1}, M Q_{n-1}, \pi_{m q}^{n-1}\right) \text { for } n \geq 3 .
$$

where, $\pi_{m q}^{n-1}$ can be determined from definition 1 in [2]. The basis for the above definition is $M Q_{2}=Q_{2}$.

A $M Q_{4}$, where $\pi_{m q}^{3}=\{0,3,2,1,4,7,6,5\}$, is shown in Fig. 5. It can be observed that from Fig. 5 that the diameter of $M Q_{4}$ is 3 as compared to $Q_{4}$ which has a diameter of 4 . It has been shown [2] that, in general the diameter of $M Q_{n}$ is $\lceil(n+1) / 2\rceil$. The properties of $M Q_{n}$ and an optimal routing strategy for exploiting the reduced diameter are discussed in [2].

\subsection{Comments}

We have shown that the $n$-cube, the twisted $n$-cube and the multiply-twisted $n$-cube can all be constructed using the construction procedure described in section 2 hence by theorem 3.8 all three of them belong to the class of HL-graphs. We have recently identified a new hypercube-like topology which is based on the recursive definition of the twisted 3cube and has a diameter of $\lceil 2 n / 3\rceil$ and other interesting properties.

\section{Routing and Broadcast Strategies 5.1 Routing strategy}

In this section we present a simple routing strategy that is applicable to all members of the class of HL-networks. 


\section{The Dimension Reducing Algorithm}

The routing strategy is presented in the form of an algorithm. Let $S$ be the source vertex and $D$ be the destination vertex.

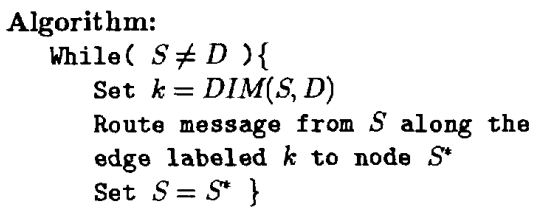

Example 5.1 Consider $T Q_{3}$ as shown in Fig. 1(b). To route a message from vertex 3 to vertex \& using the above algorithm, the steps are shown in Fig. 6. The routing is completed in 3 hops.

From example 5.1 illustrated above, we can see that the routing-algorithm does not necessarily route the message along the shortest path. However, note that after every step of routing the DIM between the new source and destination node goes on decreasing. Hence, this algorithm has been called the Dimension Reducing Algorithm.

Theorem 5.1 If $S$ is a source vertex and $D$ a destination vertex which belong to an $H L$-graph $G_{n}$ and if $\operatorname{DIM}(S, D)=k$, then the Dimension Reducing Algorithm routes from $S$ to $D$ in at most $k$ steps.

Corollary 5.1.1 For every $H L$-graph, $G_{n} \in C H L_{n}$, the diameter, $d\left(G_{n}\right)$, is less than or equal to $n$.

\subsection{Broadcast Strategy}

We present a broadcast strategy which is applicable to all HL-graphs. We show that this strategy is optimal and the broadcast completes in $n$ steps for any HLgraph $G_{n} \in$ $C H L_{n}$. The underlying assumption is that a node can send a message to only one node at any given time.

\section{The Broadcast Algorithm}

The broadcast strategy is presented in the form of an algorithm shown below.

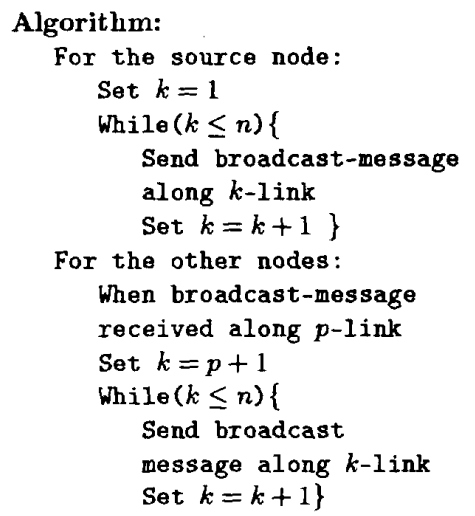

Example 5.2 The steps in a broadcast from node 5 in a $T_{3}$ are shown in Fig. 7 . The broadcast completes in 3 steps.

Theorem 5.2 For any $G_{n} \in C H L_{n}$, the broadcast algorithm is optimally terminated in n-steps.

The proof of the above theorem is based on the following observation

Observation 1 For any vertex $u$ belonging to the graph $G_{n} \in C H L_{n}$ there are exactly $2^{k-1}$ vertices having a dimension $k$ with respect to $v$.

\subsection{Comments}

In this section, we have presented a simple routing strategy for HL-networks. The routing strategy has a worst case $n$-step performance for $n$-dimensional networks. An optimal (under the assumption that any node in a HL-network can send atmost one message at a time) $n$-step broadcast algorithm has also been presented. Both these algorithms can be implemented in a distributed manner and work for any HL-network.

\section{Embedding}

It has been proved that the $n$-dimensional hypercube can embed graphs like $(n-1)$-level binary trees and arbitrary size meshes, the twisted $n$-cube can embed $n$-level binary trees. Embedding a cycle on all the vertices in a graph requires the graph to be hamiltonian. We have observed, during our studies, that all HLgraphs of dimension 2 or higher that we have come across, including the ones which do not belong to any of the known hypercube variants, are hamiltonian - the hypercube, the twisted $\mathrm{n}$-cube and the multiply twisted n-cube are known to be hamiltonian. We conjecture that all HL-graphs of dimension 2 or higher are hamiltonian. Thus, we observe that networks belonging to the class of hypercube-like graphs can embed many of important algorithmic graphs.

\section{Reconfigurability}

The class of HL-graphs is rich in terms number of members that belong to the class. By altering the permutation at one or more nodes in the binary tree that represents the construction of an HL-graph, it is possible derive one HL-graph from the other. This would amount to either a relabeling of the original HL-graph or deriving a different non-isomorphic HL-graph. Figs. 8(a) and (b) show examples of fully and partially reconfigurable 3-dimensional HL-networks.

The reconfiguration scheme proposed above preserves the labels of the nodes and of each link incident on it; the only thing that changes with respect to any particular node is a subset of its neighbours. Thus, the generalized routing and broadcast algorithms proposed earlier, in section 5 can continue to be used without modification in the reconfigured HL-network, which is a strong point in their favour. 


\section{Conclusions}

In this paper we have presented the class of hypercubelike graphs, and discussed the method of constructing such graphs. Several topological properties of this class of graphs have been discussed. It has been found that several of these properties resemble those of the binary hypercube graph. This class of graphs has a rich membership. The twisted $n$-cube and the multiply-twisted $n$-cube as well as the hypercube have been shown to belong to this class of graphs (However, the class is not limited to these alone.). A worstcase $n$-step routing strategy and a $n$-step broadcast strategy, applicable to all HL-networks, have been presented. Some properties related to emedding and schemes for achieving partial and full reconfigurability amongst HL-networks have been briefly indicated. We envisage that the class of hypercube-like graphs would be of interest to designers of multicomputer interconnection networks.

\section{Acknowledgements}

Preliminary reviews by M. J. Shankar Raman, Prof. Lawrence Jenkins and Prof. L.M. Patnaik have greatly helped in improving the quality of this paper.

\section{References}

[1] A. Esfahanian, L. M. Ni, and B. E. Sagan, "The twisted n-cube with application to multiprocessing," IEEE Transactions on Computers, vol. 40, pp. 88-93, January 1991.

21 K. Efe, "A variation on the hypercube with lower diameter," IEEE Transactions on Computers, vol. 40, pp. 1312-1316, November 1991.

[3] Y. Saad and M. H. Schultz, "Topological properties of hypercubes," IEEE Transactions on Computers, vol. 37, pp. 867872 , July 1988 .

[4] A. S. Vaidya, P. S. N. Rao, and S. R. Shankar, "A class of hypercube-like networks," Tech. Rep. EE/01/93, Dept. of Electrical Engg., Indian Institute of Science, Bangalore 560012, India, September 1993.

[5] F. Harary, Graph Theory. Reading, MA: Addison-Wesley, 1969.
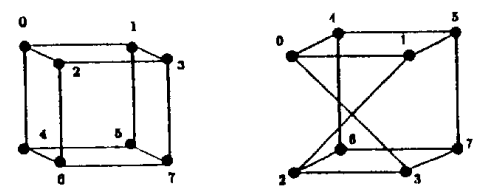

Figure 1:

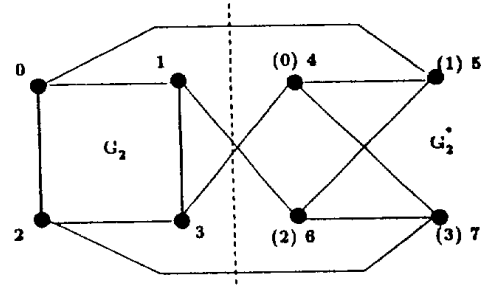

Figure 3:

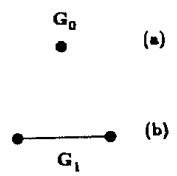

Figure 2:

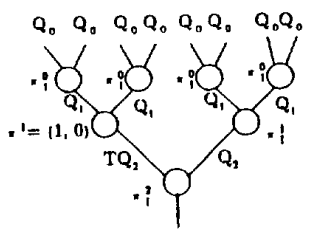

(d) Dinnry tree like representition of 70 croem-bar awitches

Figure 4:

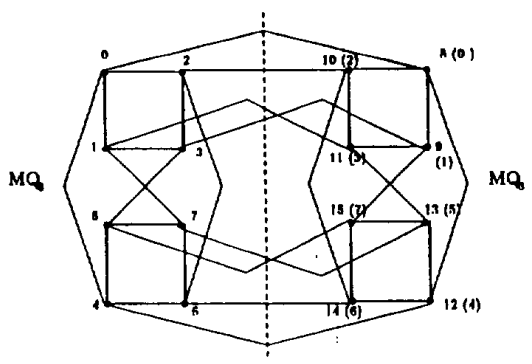

Figure 5:

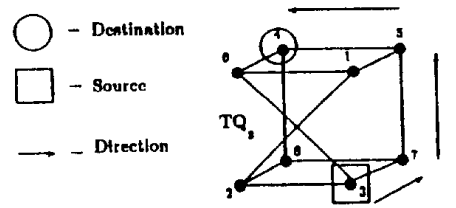

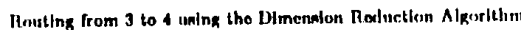
(completes in 3-hopes)

Note: Shortest path requires only 2 hope

Figure 6:
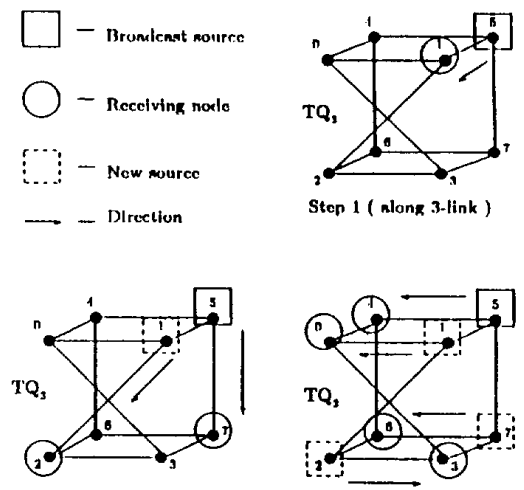

Stcp 2 ( along 2-links )

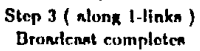

Figure 7:
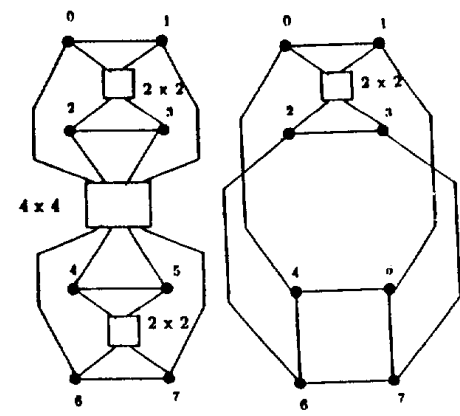

Figure 8: 